\title{
Intra-household decision-making and educational attainment in Indonesia
}

\author{
H. Haryani \& T. Dartanto \\ Department of Economics, Faculty of Economics and Business, Universitas Indonesia, Depok, Indonesia
}

\begin{abstract}
Many studies on the determinants of children's education attainment have found that parental background and family income are the most important factors. However, the current research shows the importance of intra-household decision-making on children's educational attainment. An intense discussion between parents and a child on the best path of education that the child should take, will probably have a future consequence on the child's educational attainment. This study aims at analysing the impact of children's involvement in decision-making of their educational attainment. We separate the decision-making of children's schooling choices into three types: decided by parents, decided by both parents and children, and decided by children. Which type of decision-making, regarding children's education, has the best outcome for future educational attainment? This study uses two waves of the 2000 and 2007 Indonesian Family Life Survey (IFLS) to examine this issue. Applying econometric estimations, this study confirms that the type of decision-making on children's schooling choices in 2000 has a significant effect on the future children's educational choice, and that the schooling choices decided by children has the highest impact on children's educational attainment compared to the other types of decision-making. This result suggests that parents should listen to their children in deciding their education choice.
\end{abstract}

\section{INTRODUCTION}

'Education is the most powerful weapon which you can use to change the world' (Nelson Mandela, 1918-2003)

Many studies on the determinant of children's educational attainment have found that parent's background and family income are the most important factors (Becker, 1964; Leibowitz, 1974; Becker \& Tomes, 1986; Behrman \& Rozsenzweig, 2005. However, current research shows the importance of intra-household decision-making on children's educational attainment (Fleisher, 1977; Rangel, 2006). Conventional wisdom perceives that parents are always trying to provide the best for their children (Becker, 1981; Becker \& Tomes, 1986), so that they sometimes have a dominant role in the decision of the children's schooling. The differences in parents' resources allocation for their children will cause differences in the level of achievement in children (Bloome, 2015).

Changes in environment, culture, and information have changed intra-household decision-making from a traditional type of household decision-making (parent centre) to a more democratic type of decision-making that allows all family members to actively discuss household issues (Mikkelsen, 2006; Lundberg et al., 2007). Moreover, the United Nation Convention on the Rights of the Child (1989), ratified by the Indonesian Government through Presidential Decree No. 36 (1990), stated in articles 12 \& 13 that the children shall have the right to freedom of expression. In the case of education, an intense discussion, between parents and children, about the best educational path that the child should take, would probably have optimal impact on the quantity and quality of their children's education (d'Addio, 2007), while the children would be fully responsible for the decision made by both parties. 
The study aims at analysing the impact of children's involvement in decision-making for their educational attainment. We separate the decision-making of children's schooling choices into three types: decided by parents, decided by both parents and children, and decided by children. Which type of decision-making, regarding children's education, has the best outcome for future educational attainment?

\section{THEORETICAL FRAMEWORK}

This study uses a model of collective household (Chiappori, 1992; Browning \& Chiappori, 1998), seeking to maximise the utility of family consisting of parents and children utility. Simplified, this study uses the assumption that a household consists of two parents and one child (Lundberg et al., 2007). Parents' decisions are unitary models with the assumption that they share the same preference and/or decision or only one parent makes the decision.

The utility functions of a family are formed from the variable of other commodity consumption, the quantity, and quality of children in the family (Becker, 1981). Other commodity consumption includes all family consumption like food, clothing, and housing in general. The quantity of children is defined as the number of children in a family. Furthermore, the quality of children in this study refers to the educational attainment of children.

The utility function of a family is a bundle utility of parents and their children. $U=g\left(U_{c}, U_{p}\right)$ where $\mathrm{U}_{\mathrm{c}}=\mathrm{U}_{\mathrm{c}}(\mathrm{x}, \mathrm{n}, \mathrm{q}), \mathrm{U}_{\mathrm{p}}=\mathrm{U}_{\mathrm{p}}(\mathrm{x}, \mathrm{n}, \mathrm{q})$ and $U_{c} \neq U_{p}$. The cooperative outcome is the solution to:

$$
\max U=\alpha U_{c}(x, n, q)+(1-\alpha) U_{p}(x, n, q)
$$

subject to the budget constraint:

$$
p_{x} x+p_{n} n+p_{q} q=y
$$

where, $U$ is a family utility consisting of child utility $\left(U_{c}\right)$ and parents' utility $\left(U_{p}\right), x$ is the general commodity consumption for all members of the family, $\mathrm{n}$ describes the number of children in the family, and q is usually the quality of child in the family (whereas in this study the quality of the child is described by the child's educational attainment). The income and expenditure constraint consists of the total income of the family, personal consumption, expenditures for each child, and the investment in a child's education costs. $\alpha$ is the contribution of children utility in the family utility besides parents.

Specifically, a child's quality function is:

$$
q^{*}=f\left(p_{x}^{*}, p_{n}^{*}, p_{q}^{*}, y^{*}, \alpha^{*}\right)
$$

It can be shown that the model produces standard demand functions for consumption goods, leisure, and education (a formal proof is contained in Browning and Chiappori (1998)). These functions depend on the price of education, wages, household resources, the distribution of power, and household characteristics (observable and unobservable) (Mazzocco, 2007).

To analyse the impact of children's involvement in decision-making on educational attainment, we separated the decision-making choice of school children into three types: liberal, authoritarian and democratic. Bargaining power of children within household can be observed from from the value of $\alpha$, namely:

1. If $\alpha$ value is 1 this means that the decision in education is entirely in the authority of children (liberal/freedom);

2. If $\alpha$ value is 0 this means that the decision is entirely in the authority of parents (authoritarian); 
3. If $\alpha$ value is between 1 and 0 , then the decision is made by both parents and children together (democratic).

Involving children in decision-making in education is a strategy that may be done by parents to maximise the satisfaction of the family in improving the children's educational attainment in the final education results. This happens because of communication between parents and children. Involving children in decision-making in the household regarding their education impacts the motivation of children positively because they psychologically feel the full support from their parents, so their potential will be maximal (Smart \& Pascarella, 1986; Papalia, 2004).

Based on Equation 1, an important variable that needs to be considered is the quality of the children. Thus, parents should pay attention to the quality of children in the family aside from other variables. Using Equation 3, it can be determined that decision-making affects the children $(\alpha)$ in a family and the quality of children reflected in the educational attainment of children (q). In the theory described above, decision-making in education involving the children positively affects the educational attainment of children. In other words, if the predominance of children in decision-making in education increases, the educational attainment of the children also increases. However, based on the literature, educational attainment of children is also affected by family income, family expenditure for each child (for health care, clothing, and so on, which is accumulated according to the number of children), the consumption of other commodities, and household characteristics (children and parents/ family) that are observable and unobservable (Becker, 1981; Mazzocco, 2007).

The contribution of children's utility $(\alpha)$ describes the bargaining power of children in a family and their involvement in decision-making in the family. From the conclusion above, it can be seen that if children are included in decision-making regarding the choice of their school/education, it will have a positive impact and produce better educational attainment.

\section{RESEARCH METHODOLOGY}

In our empirical analysis, we write the model to be estimated in a log-linear form:

$$
q_{i t}=\beta_{0}+\beta_{1} d m_{i t-1}+\beta_{2} h_{c i t-1}+\beta_{3} h_{p i t-1}+\epsilon_{l}
$$

where, $q_{i t}$ is the educational attainment (years of schooling) in 2007, which measures child quality, $d m_{i t-1}$ is the decision-making in the children's education in $2000, h_{c i t-1}$ is a vector of children background variables (age, number of siblings, and cognitive abilities) in $2000, h_{\text {pit-1 }}$ is a vector of parents/families background variables (the age of the head of household, families living, parents education, and families income), and $\epsilon_{i}$ is a random error term that is decided by children distributed across families but may be correlated across siblings. Table 1 shows the operational definitions of the variables used in this study:

This study uses two waves of the 2000 and 2007 Indonesian Family Life Survey (IFLS) to examine this issue. The IFLS is a comprehensive longitudinal socioeconomic survey that represents an area including 13 of Indonesia's 26 provinces and 83 percent of its population (Frankenberg \& Thomas, 2000). The IFLS contains, among other measures, detailed information on family structure and composition, marriage, school enrolment and completion, parents' employment, income of each family member, total real expenditure, and within household decision-making. IFLS has numerous strengths: nearly everyone in the household was interviewed directly so that the data is both comprehensive and largely self-reported.

To see the effect of the role of the child's education decision makers, we used the data of 2000, and the educational attainment of children who are dependent variables used 2007 data. The observations in this study were children between 11-18 years old and with unmarried status, in the household and interviewed in 2000. Table 2 shows the summary statistics, including the mean and standard deviation of each variable. These are 3,908 children between 11-18 years old in the sample. 
Table 1. Variable operational definition.

\begin{tabular}{|c|c|}
\hline Dependent variable (2007) & The meaning of the variable \\
\hline Child's education (q) & Child's years of schooling \\
\hline Explanatory variable (2000) & The meaning of the variable \\
\hline $\begin{array}{l}\text { Children involvement in decision-making on } \\
\text { children's education (decided by both parents and } \\
\text { children or only by children) (democratic) }\end{array}$ & $\begin{array}{l}\text { Dummy ( } 1 \text { if children involvement in } \\
\text { decision-making on children's education, } \\
0 \text { if not) }\end{array}$ \\
\hline $\begin{array}{l}\text { Decision-making on children's education decided } \\
\text { by both parents and children }(\mathrm{dm} 1)\end{array}$ & $\begin{array}{l}\text { Dummy ( } 1 \text { if decision-making on children's } \\
\text { education decided by both parents and } \\
\text { children, } 0 \text { if not) }\end{array}$ \\
\hline $\begin{array}{l}\text { Decision-making on children's education decided } \\
\text { by children }(\mathrm{dm} 2)\end{array}$ & $\begin{array}{l}\text { Dummy ( } 1 \text { if decision-making on children's } \\
\text { education decided by both parents and } \\
\text { children, } 0 \text { if not })\end{array}$ \\
\hline \multicolumn{2}{|l|}{ Child vector variable } \\
\hline Child's cognitive ability (c_kog00) & Child's cognitive in analysis and maths. \\
\hline Age of child (c_age 00 ) and child's age square & Age of child and child's age square \\
\hline Sex of child (c_sex 00$)$ & Dummy ( 1 if female and 0 if male) \\
\hline Child's status (c_stat00) & $\begin{array}{l}\text { Dummy ( } 1 \text { if the status of child is adopted } \\
\text { or stepchild, } 0 \text { if real child) }\end{array}$ \\
\hline Child's schooling status (c_sch00) & $\begin{array}{l}\text { Dummy ( } 1 \text { if the child is still schooling, } \\
0 \text { if not) }\end{array}$ \\
\hline \multicolumn{2}{|l|}{ Family vector variable } \\
\hline Residence of family (c_res00) & $\begin{array}{l}\text { Dummy ( } 1 \text { if living in rural, } 0 \text { if living in } \\
\text { urban) }\end{array}$ \\
\hline Number of siblings (num_sib00) & $\begin{array}{l}\text { Number of siblings, both real siblings and } \\
\text { adopted/stepsiblings }\end{array}$ \\
\hline $\begin{array}{l}\text { Age of household's head (krt_age00) and age of } \\
\text { household's head square (krt_agesq00) }\end{array}$ & $\begin{array}{l}\text { The age of household's head and household's } \\
\text { head age square }\end{array}$ \\
\hline Father's education (pf_yos00) & Father's years of schooling \\
\hline Mother's education (pm_yos00) & Mother's years of schooling \\
\hline Log total real family expenditure (lnf_rtotal00) & Log total real family expenditure \\
\hline Log total expenditure for education (lnxeducall00) & Log total expenditure for education \\
\hline
\end{tabular}

Source: Authors.

\section{ANALYSIS OF RESULTS}

In Figure 1 we can see the average educational attainment based on education decision makers. Educational decision-making by children has resulted in educational attainment that is the higher than when decided by others. The average educational attainment based on the type of decision by parent decision-making becomes the lowest kind of decision-making, compared with the two other types of decision-making. The results are consistent with the theoretical framework above.

From Table 3, it can be seen that if children are involved in decision-making regarding their educational choice (democracy) in 2000, it statistically has a positive impact on educational attainment of the children in 2007. This means that if the children are involved in decision-making regarding their education, then the educational attainment is better than if the children are excluded. The magnitude of all decision-making coefficients in education involving children (democracy) has the same direction and shows consistent results. These results are consistent with the theoretical framework that has been presented in the previous section. 
Table 2. Statistics summary.

\begin{tabular}{|c|c|c|c|c|c|}
\hline Variable & Obs & Mean & Std. Dev. & Min & Max \\
\hline Child's education in 2007 (c_yos07) & 3,908 & 10.385 & 3.298 & 0 & 18 \\
\hline $\begin{array}{l}\text { Decision-making on children's education } \\
\text { decided by parents and children (dm1) }\end{array}$ & 3,908 & 0.099 & 0.299 & 0 & 1 \\
\hline $\begin{array}{l}\text { Decision-making on children's education } \\
\text { decided by children (dm2) }\end{array}$ & 3,908 & 0.018 & 0.134 & 0 & 1 \\
\hline $\begin{array}{l}\text { Decision-making on children's education } \\
\text { decided by parents (dm3) }\end{array}$ & 3,908 & 0.883 & 0.322 & 0 & 1 \\
\hline $\begin{array}{l}\text { Decision-making on children's education } \\
\text { (c_dm) }\end{array}$ & 3,908 & 2.783 & 0.607 & 1 & 3 \\
\hline $\begin{array}{l}\text { Children's involvement in decision-making } \\
\text { on children's education (decided by parents } \\
\text { and children or decided by only children) } \\
\text { (demokrasi) }\end{array}$ & 3,908 & 0.117 & 0.322 & 0 & 1 \\
\hline Child's education in 2000 (c_yos00) & 3,908 & 7.031 & 2.515 & 0 & 16 \\
\hline Child's cognitive ability (c_kog00) & 3,908 & 63.619 & 26.799 & 0 & 100 \\
\hline Age of child (c_age 00 ) & 3,908 & 14.353 & 2.253 & 11 & 18 \\
\hline Child's age square (c_agesq00) & 3,908 & 211.093 & 65.171 & 121 & 324 \\
\hline Sex of child (c_sex00) & 3,908 & 0.478 & 0.499 & 0 & 1 \\
\hline Child's status (c_stat00) & 3,908 & 0.024 & 0.154 & 0 & 1 \\
\hline Child's schooling status (c_school07) & 3,908 & 0.131 & 0.337 & 0 & 1 \\
\hline Residence of family (c_res00) & 3,908 & 0.549 & 0.498 & 0 & 1 \\
\hline Number of siblings (num_sib00) & 3,908 & 3.990 & 1.758 & 1 & 11 \\
\hline Age of head household (krt_age00) & 3,908 & 46.190 & 8.256 & 25 & 105 \\
\hline Age of head household square (krt_age00) & 3,908 & $2,191.053$ & 817.667 & 625 & 11,025 \\
\hline Father's education (pf_yos00) & 3,908 & 6.343 & 4.329 & 0 & 18 \\
\hline Mother's education (pm_yos00) & 3,908 & 5.094 & 3.971 & 0 & 17 \\
\hline $\begin{array}{l}\text { Log total real family expenditure } \\
\text { (lnf_rtotal00) }\end{array}$ & 3,908 & 13.929 & 0.663 & 11.082 & 16.607 \\
\hline $\begin{array}{l}\text { Log total expenditure for education } \\
\text { (lnxeducallo0) }\end{array}$ & 3,717 & 11.056 & 1.154 & 5.809 & 15.093 \\
\hline
\end{tabular}

Source: IFLS, authors.

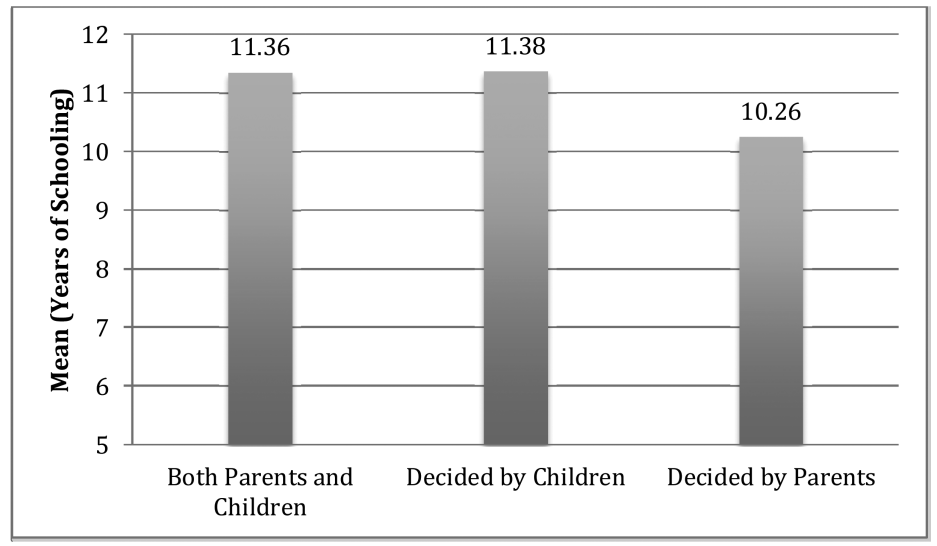

Figure 1. The type of decision-making and years of schooling.

Furthermore, to obtain in-depth and detailed information, the separation of decision makers in education is divided into two dummy variables, namely decision-making in education decided jointly by the children and parents $(\mathrm{dm} 1)$ and decision-making in education decided by children alone $(\mathrm{dm} 2)$. Decision-making in education by parents is used as a basis group. 
Table 3. Regression result with 1 dummy variable of decision-making.

\begin{tabular}{|c|c|c|c|c|c|c|}
\hline \multirow[b]{3}{*}{ Explanatory variables } & \multicolumn{6}{|c|}{$\begin{array}{l}\text { Dependent variable: Child's educational attainment on } 2007 \text { (years of } \\
\text { schooling) (c_yos07) }\end{array}$} \\
\hline & \multicolumn{2}{|c|}{ Child's age $11-18$ years } & \multicolumn{2}{|c|}{ Child's age $11-14$ years } & \multicolumn{2}{|c|}{ Child's age $15-18$ years } \\
\hline & Model 1 & Model 2 & Model 1 & Model 2 & Model 1 & Model 2 \\
\hline $\begin{array}{l}\text { Children's involvement } \\
\text { in decision-making } \\
\text { on their education } \\
\text { attainment }\end{array}$ & $\begin{array}{l}0.4942^{* * *} \\
(0.130)\end{array}$ & $\begin{array}{l}0.4396^{* * * *} \\
(0.114\end{array}$ & $\begin{array}{l}0.4271^{* * *} \\
(0.166)\end{array}$ & $\begin{array}{l}0.3093^{* * *} \\
(0.160)\end{array}$ & $\begin{array}{l}0.5120^{* * *} \\
(0.168)\end{array}$ & $\begin{array}{l}0.5349 * * * \\
(0.156)\end{array}$ \\
\hline $\begin{array}{l}\text { Decision-making in } \\
\text { children's education } \\
\text { decided by parents } \\
\text { and children }(\mathrm{dm} 1)\end{array}$ & ---- & ---- & ---- & ---- & ---- & ---- \\
\hline $\begin{array}{l}\text { Decision-making in } \\
\text { children's education } \\
\text { decided by parents } \\
\text { and children }(\mathrm{dm} 2)\end{array}$ & ----- & ----- & ----- & ----- & ----- & ----- \\
\hline $\begin{array}{l}\text { Children's cognitive } \\
\text { ability (c_kog00) }\end{array}$ & $\begin{array}{l}0.0293 * * * \\
(0.002)\end{array}$ & $\begin{array}{l}0.0251 * * * \\
(0.002)\end{array}$ & $\begin{array}{l}0.0320^{* * *} \\
(0.003)\end{array}$ & $\begin{array}{l}0.0280^{* * * *} \\
(0.003)\end{array}$ & $\begin{array}{l}0.0282^{* * *} \\
(0.002)\end{array}$ & $\begin{array}{l}0.0237 * * * \\
(0.002)\end{array}$ \\
\hline $\begin{array}{l}\text { Age of children } \\
\text { (c_age } 00)\end{array}$ & $\begin{array}{l}1.1554 * * * \\
(0.261)\end{array}$ & $\begin{array}{l}0.8676^{* * * *} \\
(0.256)\end{array}$ & $\begin{array}{l}3.6298 * * * \\
(1.3233)\end{array}$ & $\begin{array}{l}2.8973^{* *} \\
(1.287)\end{array}$ & $\begin{array}{l}1.3361 \\
(1.989)\end{array}$ & $\begin{array}{l}-0.0461 \\
(1.960)\end{array}$ \\
\hline $\begin{array}{l}\text { Age of child square } \\
\text { (c_agesq00) }\end{array}$ & $\begin{array}{l}-0.0323^{* * *} \\
(0.009)\end{array}$ & $\begin{array}{l}-0.0228^{* *} \\
(0.009)\end{array}$ & $\begin{array}{l}-0.1349^{* *} \\
(0.053)\end{array}$ & $\begin{array}{l}-0.1060^{* *} \\
(0.051)\end{array}$ & $\begin{array}{l}-0.0404 \\
(0.060)\end{array}$ & $\begin{array}{l}0.0022 \\
(0.060)\end{array}$ \\
\hline $\begin{array}{l}\text { Sex of children } \\
\text { (c_sex00) }\end{array}$ & $\begin{array}{l}0.3393 * * * \\
(0.080)\end{array}$ & $\begin{array}{l}0.3377 * * * \\
(0.078)\end{array}$ & $\begin{array}{l}0.1967^{*} \\
(0.107)\end{array}$ & $\begin{array}{l}0.2072 * * \\
(0.103)\end{array}$ & $\begin{array}{l}0.4890 * * * \\
(0.121)\end{array}$ & $\begin{array}{l}0.4895^{* * *} \\
(0.119)\end{array}$ \\
\hline \multirow[t]{3}{*}{$\begin{array}{l}\text { Children's status } \\
\text { (c_stat00) }\end{array}$} & $\begin{array}{l}-0.4016 \\
(0.257)\end{array}$ & $\begin{array}{l}-0.2876 \\
(0.242)\end{array}$ & $\begin{array}{l}-0.6915^{* *} \\
(0.344)\end{array}$ & $\begin{array}{l}-0.3561 \\
(0.337)\end{array}$ & $\begin{array}{l}-0.0757 \\
(0.381)\end{array}$ & $\begin{array}{l}-0.1298 \\
(0.356)\end{array}$ \\
\hline & \multicolumn{2}{|c|}{ Child's age $11-18$ years } & \multicolumn{2}{|c|}{ Child's age $11-14$ years } & \multicolumn{2}{|c|}{ Child's age $15-18$ years } \\
\hline & Model 3 & Model 4 & Model 3 & Model 4 & Model 3 & Model 4 \\
\hline $\begin{array}{l}\text { Children's schooling } \\
\text { status (c_school07) }\end{array}$ & $\begin{array}{l}1.3149 * * * \\
(0.117)\end{array}$ & $\begin{array}{l}1.2830^{* * *} \\
(0.114)\end{array}$ & $\begin{array}{l}1.4233^{* * *} \\
(0.126)\end{array}$ & $\begin{array}{l}1.3571 * * * \\
(0.125)\end{array}$ & $\begin{array}{l}1.4873^{* * *} \\
(0.288)\end{array}$ & $\begin{array}{l}1.3696^{* * *} \\
(0.270)\end{array}$ \\
\hline $\begin{array}{l}\text { Residence of family } \\
\text { (c_res00) }\end{array}$ & $\begin{array}{l}-0.3165^{* * *} \\
(0.086)\end{array}$ & $\begin{array}{l}-0.0626 \\
(0.086)\end{array}$ & $\begin{array}{l}-0.2151^{*} \\
(0.117)\end{array}$ & $\begin{array}{l}0.0253 \\
(0.116)\end{array}$ & $\begin{array}{l}-0.4189 * * * \\
(0.128)\end{array}$ & $\begin{array}{l}-0.1849 \\
(0.128)\end{array}$ \\
\hline $\begin{array}{l}\text { Number of siblings } \\
\text { (num_sib00) }\end{array}$ & $\begin{array}{l}-0.1604 * * * \\
(0.025)\end{array}$ & $\begin{array}{l}-0.2299^{* * *} \\
(0.024)\end{array}$ & $\begin{array}{l}-0.1102^{* * *} \\
(0.035)\end{array}$ & $\begin{array}{l}-0.1664 * * * \\
(0.034)\end{array}$ & $\begin{array}{l}-0.2154^{* * *} \\
(0.036)\end{array}$ & $\begin{array}{l}-0.2895^{* * *} \\
(0.035)\end{array}$ \\
\hline $\begin{array}{l}\text { Age of household head } \\
\quad \text { (krt_age00) }\end{array}$ & $\begin{array}{l}0.0374 \\
(0.042)\end{array}$ & $\begin{array}{l}0.0354 \\
(0.045)\end{array}$ & $\begin{array}{l}0.0071 \\
(0.055)\end{array}$ & $\begin{array}{l}-0.0266 \\
(0.053)\end{array}$ & $\begin{array}{l}0.0719 \\
(0.070)\end{array}$ & $\begin{array}{l}0.1868 * * \\
(0.081)\end{array}$ \\
\hline $\begin{array}{l}\text { Age of household head } \\
\text { square (krt_agesq00) }\end{array}$ & $\begin{array}{l}-0.0003 \\
(0.0004)\end{array}$ & $\begin{array}{l}-0.0002 \\
(0.0004)\end{array}$ & $\begin{array}{l}-0.0004 \\
(0.0005)\end{array}$ & $\begin{array}{l}-0.0003 \\
(0.0005)\end{array}$ & $\begin{array}{l}-0.0005 \\
(0.0007)\end{array}$ & $\begin{array}{l}-0.0015^{*} \\
(0.0008)\end{array}$ \\
\hline $\begin{array}{l}\text { Father's education } \\
\text { (pf_yos00) }\end{array}$ & $\begin{array}{l}0.1856^{* * * *} \\
(0.013)\end{array}$ & $\begin{array}{l}0.1512 * * * \\
(0.013)\end{array}$ & $\begin{array}{l}0.1804^{* * * *} \\
(0.018)\end{array}$ & $\begin{array}{l}0.1547 * * * \\
(0.017)\end{array}$ & $\begin{array}{l}0.1852^{* * * *} \\
(0.019)\end{array}$ & $\begin{array}{l}0.1396 * * * \\
(0.019)\end{array}$ \\
\hline $\begin{array}{l}\text { Mother's education } \\
\text { (pm_yos00) }\end{array}$ & $\begin{array}{l}0.1272 * * * \\
(0.014)\end{array}$ & $\begin{array}{l}0.1031 * * * \\
(0.014)\end{array}$ & $\begin{array}{l}0.1033^{* * * *} \\
(0.019)\end{array}$ & $\begin{array}{l}0.0818^{* * *} \\
(0.018)\end{array}$ & $\begin{array}{l}0.1508^{* * *} \\
(0.021)\end{array}$ & $\begin{array}{l}0.1204 * * * \\
(0.020)\end{array}$ \\
\hline $\begin{array}{l}\text { Log total real family } \\
\text { expenditure } \\
\text { (lnf_rtotal00) }\end{array}$ & $\begin{array}{l}0.6413^{* * *} \\
(0.075)\end{array}$ & ----- & $\begin{array}{l}0.3561^{* * * *} \\
(0.102)\end{array}$ & ----- & $\begin{array}{l}0.9250^{* * * *} \\
(0.109)\end{array}$ & ----- \\
\hline $\begin{array}{l}\text { Log total expenditure } \\
\text { for education } \\
\text { (lnxeducallo0) }\end{array}$ & ----- & $\begin{array}{l}0.7538^{* * * *} \\
(0.043)\end{array}$ & ----- & $\begin{array}{l}0.6215^{* * *} \\
(0.057)\end{array}$ & ----- & $\begin{array}{l}0.8999 * * * \\
(0.065)\end{array}$ \\
\hline Constant & $\begin{array}{l}-12.686^{* * *} \\
(2.251)\end{array}$ & $\begin{array}{l}-9.239 * * * \\
(2.052)\end{array}$ & $\begin{array}{l}-22.901 \\
(8.299)\end{array}$ & $\begin{array}{l}-18.670 * * \\
(7.971)\end{array}$ & $\begin{array}{l}-18.292 \\
(16.414)\end{array}$ & $\begin{array}{l}-6.463 \\
(16.223)\end{array}$ \\
\hline F-Statistic & 227.63 & 239.33 & 113.47 & 123.16 & 122.71 & 123.59 \\
\hline R-Squared & 0.4294 & 0.4671 & 0.4112 & 0.4412 & 0.4543 & 0.4917 \\
\hline \# of observations & 3,908 & 3,717 & 2,071 & 2,030 & 1,837 & 1,687 \\
\hline
\end{tabular}

Note: $\mathrm{t}$-statistics are in parentheses: ${ }^{* * *} \mathrm{p}<0.01,{ }^{* *} \mathrm{p}<0.05,{ }^{*} \mathrm{p}<0.1$.

Source: authors. 
Table 4. Regression result with 2 dummy variables of decision-making.

\begin{tabular}{|c|c|c|c|c|c|c|}
\hline \multirow[b]{3}{*}{ Explanatory variables } & \multicolumn{6}{|c|}{$\begin{array}{l}\text { Dependent variable: Child's educational attainment on } 2007 \text { (years } \\
\text { of schooling) (c_yos07) }\end{array}$} \\
\hline & \multicolumn{2}{|c|}{ Child's age $11-18$ years } & \multicolumn{2}{|c|}{ Child's age $11-14$ years } & \multicolumn{2}{|c|}{ Child's age $15-18$ years } \\
\hline & Model 3 & Model 4 & Model 3 & Model 4 & Model 3 & Model 4 \\
\hline $\begin{array}{l}\text { Children's involvement } \\
\text { in decision-making } \\
\text { on their education } \\
\text { attainment }\end{array}$ & ----- & ----- & ----- & ----- & ----- & ----- \\
\hline $\begin{array}{l}\text { Decision-making in } \\
\text { children's education } \\
\text { decided by parents } \\
\text { and children }(\mathrm{dm} 1)\end{array}$ & $\begin{array}{l}0.4898 * * * \\
(0.130)\end{array}$ & $\begin{array}{l}0.3917 * * * \\
(0.123)\end{array}$ & $\begin{array}{l}0.4045^{* *} \\
(0.182)\end{array}$ & $\begin{array}{l}0.2601 \\
(0.174)\end{array}$ & $\begin{array}{l}0.5396 * * * \\
(0.181)\end{array}$ & $\begin{array}{l}0.4904 * * * \\
(0.169)\end{array}$ \\
\hline $\begin{array}{l}\text { Decision-making in } \\
\text { children's education } \\
\text { decided by parents } \\
\text { and children }(\mathrm{dm} 2)\end{array}$ & $\begin{array}{l}0.5187^{*} \\
(0.272)\end{array}$ & $\begin{array}{l}0.7088 * * * \\
(0.258)\end{array}$ & $\begin{array}{l}0.5546 \\
(0.358)\end{array}$ & $\begin{array}{l}0.5943^{*} \\
(0.353)\end{array}$ & $\begin{array}{l}0.3619 \\
(0.389)\end{array}$ & $\begin{array}{l}0.7790^{* *} \\
(0.356)\end{array}$ \\
\hline $\begin{array}{l}\text { Children cognitive } \\
\text { ability (c_kog00) }\end{array}$ & $\begin{array}{l}0.0293 * * * \\
(0.002)\end{array}$ & $\begin{array}{l}0.0251 * * * \\
(0.002)\end{array}$ & $\begin{array}{l}0.0320 * * * \\
(0.003)\end{array}$ & $\begin{array}{l}0.0280 * * * \\
(0.003)\end{array}$ & $\begin{array}{l}0.0282 * * * \\
(0.002)\end{array}$ & $\begin{array}{l}0.0237 * * * \\
(0.002)\end{array}$ \\
\hline $\begin{array}{l}\text { Age of children } \\
\text { (c_age00) }\end{array}$ & $\begin{array}{l}1.1548 * * * \\
(0.261)\end{array}$ & $\begin{array}{l}0.8609 * * * \\
(0.256)\end{array}$ & $\begin{array}{l}3.6456^{* * *} \\
(1.3255)\end{array}$ & $\begin{array}{l}2.9295^{* *} \\
(1.288)\end{array}$ & $\begin{array}{l}1.3570 \\
(1.991)\end{array}$ & $\begin{array}{l}-0.0733 \\
(1.962)\end{array}$ \\
\hline $\begin{array}{l}\text { Age of child square } \\
\text { (c_agesq00) }\end{array}$ & $\begin{array}{l}-0.0323 * * * \\
(0.009)\end{array}$ & $\begin{array}{l}-0.0225^{* *} \\
(0.009)\end{array}$ & $\begin{array}{l}-0.1356^{* *} \\
(0.053)\end{array}$ & $\begin{array}{l}-0.1073^{* *} \\
(0.052)\end{array}$ & $\begin{array}{l}-0.0410 \\
(0.060)\end{array}$ & $\begin{array}{l}0.0031 \\
(0.060)\end{array}$ \\
\hline $\begin{array}{l}\text { Sex of children } \\
\quad(\text { c_sex } 00)\end{array}$ & $\begin{array}{l}0.3393^{* * * *} \\
(0.080)\end{array}$ & $\begin{array}{l}0.3382 * * * \\
(0.078)\end{array}$ & $\begin{array}{l}0.1967 * \\
(0.107)\end{array}$ & $\begin{array}{l}0.2067 * * \\
(0.103)\end{array}$ & $\begin{array}{l}0.4879 * * * \\
(0.121)\end{array}$ & $\begin{array}{l}0.4913^{* * * *} \\
(0.119)\end{array}$ \\
\hline $\begin{array}{l}\text { Children's status } \\
\text { (c_stat00) }\end{array}$ & $\begin{array}{l}-0.4014 \\
(0.257)\end{array}$ & $\begin{array}{l}-0.2850 \\
(0.242)\end{array}$ & $\begin{array}{l}-0.6896^{* *} \\
(0.344)\end{array}$ & $\begin{array}{l}-0.3514 \\
(0.338)\end{array}$ & $\begin{array}{l}-0.0763 \\
(0.381)\end{array}$ & $\begin{array}{l}-0.1280 \\
(0.356)\end{array}$ \\
\hline $\begin{array}{l}\text { Children's schooling } \\
\text { status (c_school07) }\end{array}$ & $\begin{array}{l}1.3147^{* * * *} \\
(0.116)\end{array}$ & $\begin{array}{l}1.2813^{* * * *} \\
(0.114)\end{array}$ & $\begin{array}{l}1.4337^{* * *} \\
(0.126)\end{array}$ & $\begin{array}{l}1.3566^{* * * *} \\
(0.125)\end{array}$ & $\begin{array}{l}1.4893^{* * * *} \\
(0.288)\end{array}$ & $\begin{array}{l}1.3651^{* * *} \\
(0.270)\end{array}$ \\
\hline $\begin{array}{l}\text { Residence of family } \\
\quad \text { (c_res00) }\end{array}$ & $\begin{array}{l}-0.3165^{* * * *} \\
(0.086)\end{array}$ & $\begin{array}{l}-0.0613 \\
(0.086)\end{array}$ & $\begin{array}{l}-0.2158^{*} \\
(0.117)\end{array}$ & $\begin{array}{l}0.0243 \\
(0.116)\end{array}$ & $\begin{array}{l}-0.4300^{* * *} \\
(0.128)\end{array}$ & $\begin{array}{l}-0.1814 \\
(0.128)\end{array}$ \\
\hline $\begin{array}{l}\text { Number of siblings } \\
\text { (num_sib00) }\end{array}$ & $\begin{array}{l}-0.1604 * * * \\
(0.025)\end{array}$ & $\begin{array}{l}-0.2297 * * * \\
(0.024)\end{array}$ & $\begin{array}{l}-0.1098 * * * \\
(0.035)\end{array}$ & $\begin{array}{l}-0.1657 * * * \\
(0.034)\end{array}$ & $\begin{array}{l}-0.2154^{* * *} \\
(0.036)\end{array}$ & $\begin{array}{l}-0.2896^{* * * *} \\
(0.035)\end{array}$ \\
\hline $\begin{array}{l}\text { Age of household head } \\
\text { (krt_age00) }\end{array}$ & $\begin{array}{l}0.0373 \\
(0.042)\end{array}$ & $\begin{array}{l}0.0342 \\
(0.045)\end{array}$ & $\begin{array}{l}0.0066 \\
(0.055)\end{array}$ & $\begin{array}{l}-0.0279 \\
(0.053)\end{array}$ & $\begin{array}{l}0.0725 \\
(0.070)\end{array}$ & $\begin{array}{l}0.1855^{* *} \\
(0.081)\end{array}$ \\
\hline $\begin{array}{l}\text { Age of household head } \\
\text { square (krt_agesq00) }\end{array}$ & $\begin{array}{l}-0.0003 \\
(0.0004)\end{array}$ & $\begin{array}{l}-0.0002 \\
(0.0004)\end{array}$ & $\begin{array}{l}-0.0004 \\
(0.0005)\end{array}$ & $\begin{array}{l}-0.0003 \\
(0.0005)\end{array}$ & $\begin{array}{l}-0.0005 \\
(0.0007)\end{array}$ & $\begin{array}{l}-0.0015^{*} \\
(0.0008)\end{array}$ \\
\hline $\begin{array}{l}\text { Father's education } \\
\quad\left(\mathrm{pf} \_ \text {yos } 00\right)\end{array}$ & $\begin{array}{l}0.1856^{* * * *} \\
(0.013)\end{array}$ & $\begin{array}{l}0.1514 * * * \\
(0.013)\end{array}$ & $\begin{array}{l}0.1805^{* * * *} \\
(0.018)\end{array}$ & $\begin{array}{l}0.1548 * * * \\
(0.017)\end{array}$ & $\begin{array}{l}0.1848 * * * \\
(0.019)\end{array}$ & $\begin{array}{l}0.1398^{* * *} \\
(0.019)\end{array}$ \\
\hline $\begin{array}{l}\text { Mother's education } \\
\text { (pm_yos00) }\end{array}$ & $\begin{array}{l}0.1272^{* * *} \\
(0.014)\end{array}$ & $\begin{array}{l}0.1027 * * * \\
(0.014)\end{array}$ & $\begin{array}{l}0.1033^{* * * *} \\
(0.019)\end{array}$ & $\begin{array}{l}0.0817 * * * \\
(0.018)\end{array}$ & $\begin{array}{l}0.1511^{* * *} \\
(0.021)\end{array}$ & $\begin{array}{l}0.1197^{* * *} * \\
(0.020)\end{array}$ \\
\hline $\begin{array}{l}\text { Log total real family } \\
\text { expenditure } \\
\text { (lnf_rtotal00) }\end{array}$ & $\begin{array}{l}0.6412^{* * * *} \\
(0.075)\end{array}$ & ---- & $\begin{array}{l}0.3566^{* * *} \\
(0.101)\end{array}$ & ---- & $\begin{array}{l}0.9264 * * * \\
(0.109)\end{array}$ & ----- \\
\hline $\begin{array}{l}\text { Log total expenditure } \\
\text { for Education } \\
(\text { Inxeducallo0) }\end{array}$ & ----- & $\begin{array}{l}0.7555^{* * * *} \\
(0.043)\end{array}$ & ----- & $\begin{array}{l}0.6214 * * * \\
(0.057)\end{array}$ & ----- & $\begin{array}{l}0.9022 * * * \\
(0.065)\end{array}$ \\
\hline Constant & $\begin{array}{l}-12.6791 * * * \\
(2.254)\end{array}$ & $\begin{array}{l}-9.1822^{* * * *} \\
(2.054)\end{array}$ & $\begin{array}{l}-22.9907 * * * \\
(8.308)\end{array}$ & $\begin{array}{l}-18.8482 * * \\
(7.978)\end{array}$ & $\begin{array}{l}-18.4956 \\
(16.444)\end{array}$ & $\begin{array}{l}-6.2390 \\
(16.240)\end{array}$ \\
\hline F-Statistic & 212.43 & 223.47 & 105.92 & 114.96 & 1148 & 115.52 \\
\hline R-Squared & 0.4294 & 0.4673 & 0.4112 & 0.4413 & 0.4544 & 0.4919 \\
\hline \# of observations & 3,908 & 3,717 & 2,071 & 2,030 & 1,837 & 1,687 \\
\hline
\end{tabular}

Note: t-statistics are in parentheses: $* * * \mathrm{p}<0.01, * * \mathrm{p}<0.05, * \mathrm{p}<0.1$.

Source: authors. 
The regression results in Table 4 show that decision-making in education is decided jointly by parents and children $(\mathrm{dm} 1)$ and if it is decided by the children alone $(\mathrm{dm} 2)$, it statistically has a significant positive impact on the educational attainment of the children when compared with the basis (decision-making decided by parents).

The division of age groups is required to view the role of decision maker for both age groups more specifically. Parents are still in control of the decision-making in education, where the children may be considered not able to determine the best option for their education. Whereas with children's age, parents increasingly give trust and listen to the children's voice by involving children in decision-making in education. In fact, parents fully entrust the decision-making of children's education to the children.

\subsection{Decision-making on children's education decided by parents and children}

Based on Table 4, it can be seen that the decision-making in education decided solely by children $(\mathrm{dm} 2)$ in the children's schooling choice in 2000 produces better educational attainment than the other types of decision-making in education in 2007. It is suggested that this type of decision-making produces the highest educational attainment compared to other types of decision-making. Overall the regression results still show consistent results with the theory presented in the previous section.

Decision-making in children's education that is handed over to the children in question may have an impact on the release of their fullest potential. Children who are able to decide and choose their own school/education are identified as children who have a more mature and positive self-concept. According to Smart and Pascarella (1986), the children who have a mature and positive self-concept know their capabilities, purposes and the direction of their life, including the capability to decide the best school/education for themselves. Thus, these children will be responsible for the decisions they take (Papalia, 2004).

The results are consistent across all specifications. The coefficients for the impact of children's involvement on decision-making on their educational attainment are positive. This implies that more bargaining power to the children is associated with the highest educational attainment. In the case of the decision-making on children's education choice decided only by children, this has the highest impact on children's educational attainment. This implies that the distribution of power within the household has to affect the children's educational attainment in the household. It is mainly affected by variables other than the distribution of power within the household, especially the age of children (Lundberg et al., 2007).

\subsection{The results of control variable}

Additional information on the result of this study is the characteristics of children and the characteristics of parents or family that have different impacts for each category to educational attainment. The variables of children's cognitive abilities, children's age, children with female sex, head household's age, paternal education (both father and mother), the total real expenditure of family, and the total education expenditure, have a positive impact on educational attainment of children. This means that as more the cognitive abilities of children increase, so more will increase the educational attainment of children. Although, there are other capabilities that could affect educational attainment in addition to the cognitive abilities, such as emotional ability, and motivation of the children to learn (Pajares, 1996).

By increasing the age of children, educational attainment of children should also increase. However, along with the increasing age of children, educational attainment of children tends to stagnate. This could be because the children are no longer continuing their education, or they are already at the peak of the highest formal educational attainment. This fact also

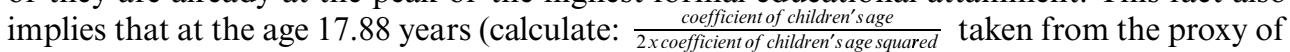
log total real expenditure of family) the average children in Indonesia quit or do not continue their education. It is most likely that at that age, the children have graduated to the level of high school (SMA-Sekolah MenengahAtas or in English Senior High School). 
Another implication to Indonesia is that 17.88 years is an age at which it is effective to ask the children to discuss their education, or even hand to over the decision in education to the children independently. These results also reflect that the decision-making in children's education is still dominated by the parents, so that the parents begin to listen to the children's voice at the age of 17.88 years; where at this age, children may have graduated from high school.

Furthermore, the higher education of the father and mother has an impact on the higher attention to children's education, so that children's education is also increased (Gang \& Zimmerman, 2000; Maralani, 2008). Likewise, family income has a positive impact on the educational attainment of children. It means that with the increasing income of a family, the greater the allocation of finance to their children's education, which affects the increasing education attainment of the children (Becker \& Tomes, 1986; Haveman \& Wolfe, 1995). Another interesting thing is that female children get a larger portion than male children, so that female children get better opportunities in educational attainment.

The number of siblings of children in a family, the children's residence, and children with the status of adopted/step children negatively affect the educational attainment of children. This study confirms a previous study which states that the number of children in a family negatively affects the children's educational attainment (Maralani, 2004). This means that by the increasing number of children living in a family, the chances of a child to study will decrease (Maralani, 2004). Likewise, children who live in a rural area have a limitation to increase their formal education, encountering a variety of obstacles (Maralani, 2008). Furthermore, the adopted/step children are included in a marginalised group, for which their education is given less attention by their adopted/step parents.

\section{CONCLUDING REMARKS}

Applying econometric estimations, this study confirms that the type of decision-making on children's schooling choices in 2000 has a significant and positive effect on the future children's educational attainment in 2007. The type of decision-making on children's education choice, decided only by children, has the highest impact on children's educational attainment compared to the other types of decision-making. The decision-making decided by children or decided by both parents and children will be made based on the age of children. These results also prove that in the second generation, the children are more concerned about the achievements of their education. This result suggests that parents should hear their children's voice when deciding their educational choice.

\section{ACKNOWLEDGEMENT}

This research is a part of Haryani's master thesis under the supervision of Teguh Dartanto, PhD The author would like to thank the generous financial support of PITTA GrantUniversitas Indonesia.

\section{REFERENCES}

Baumrind, D. (1966). Effects of authoritative parental control on child behavior. Child Development, 37(4), 887-907.

Becker, G.S. \& Tomes, N. (1986). Human capital and the rise and fall of families. Journal of Labor Economic, 4, S1-S39.

Becker, G.S. (1964). Human capital. New York: Columbia University Press.

Becker, G.S. (1981). A Treatise on the family. Cambridge: Harvard University Press.

Becker, G.S., Murphy, K \& Tamura, R. (1990). Human capital, fertility, and economic growth. Journal of Political Economy, 98, S12-S37.

Behrman, J.R. \& Rosenzweig, M.R. (2005). Does increasing women's schooling raise the schooling of the next generation? American Economic Review, 95, 1745-1751. 
Behrman, J.R. (1997). Intrahousehold distribution and the family. In M.R. Rosenzweig \& O. Stark (Eds.), Handbook of Population and Family Economics (pp. 125-187). Amsterdam: Elsevier.

Behrman, J.R., Foster, A.D., Rosenweig, M.R. \& Vashishtha, P. (1999). Women's schooling, home teaching and economic growth. Journal of Political Economy, 107, 682-714.

Bloome, D. (2015). Income inequality and intergenerational income mobility in the United States. Social Forces March, 93(3), 1047-1080.

Browning, M. \& Chiappori, P.A. (1998). Efficient intra-household allocations: A general characterization and empirical tests. Econometrica, 66,1241-1278.

Chiappori, P.A. (1992). Collective labour supply and welfare. Journal of Political Economy, 100(3), 437-467.

Currie, J. \& Moretti, E. (2003). Mother's education and the intergenerational transmission of human capital. Evidence from college openings. Quarterly Journal of Economics, 118(4), 1495-1532.

d'Addio, A.C. (2007). Intergenerational transmission of disadvantage: mobility or immobility across generations? OECD Working Paper, 52.

Ermisch, J. \& Francesconi, M. (2001). Impact of family background on educational attainments. Economica, 68(270), 137-156.

Fleisher, B.M. (1977). Mother's home time and the production of child quality. Demography, 12(2),197-212.

Frankenberg, E. \& Thomas, D. (2000). The Indonesia family life survey (IFLS): Study design and results from waves 1 and 2. Santa Monica: CA: RAND. CA. DRU-2238/1-NIA/NICHD.

Gang, I.N. \& Zimmermann, K. (2000). Is child like parent? Educational attainment and ethnic origin. The Journal of Human Resources, 35(5) 550-569.

Hamilton, L.C. (2013). Statistics with STATA: Updated for Version 12. (8thed.). Boston, USA: Brookc/ Cole, Cengage Learning.

Hanushek, E.A. \& Kimko, D.D. (2000). Schooling, labor-force quality, and the growth of nations. The American Economic Review, 90(5), 1184-1208.

Haveman, R. \& Wolfe, B. (1995). The determinants of children's attainments: A review of methods and findings. Journal of Economy Literature, 33(4) 1829-1878.

Holmlund, H., Lindahl, M. \& Plug, E. (2008). The causal effect of parent's schooling on children's schooling: A comparison of estimation methods. IZA Discussion Paper Series, 3630.

Leibowitz, A. (1974). Home investment in children. In Economics of the family: Marriage, children, and human capital, by T.W. Schultz (pp. 432-456). UMI.

Lundberg, S., Romich, J. \& Tsang, K.P. (2007). Decision-Making by children. IZA DP No. 2952. Discussion Paper Series.

Maralani, V. (2004). Family size and educational attainment in Indonesia: A cohort perspective. California Center for Population Research, CCRP 017-04.

Maralani, V. (2008). The changing relationship between family size and educational attainment over course of socioeconomic development: Evidence from Indonesia. Demography, 45(2), 693-717.

Mazzocco, M. (2007). Household intertemporal behavior: A collective characterization and a test of commitment. Review of Economic Studies, 74(3), 857-895.

Mikkelsen, M.R. (2006). Children's influence on family decision-making in food buying and consumption. Denmark: The National Institute of Public Health Denmark and MAPP-Centre for Research on Customer Relations in the Food Sector. Aarhus School of Business Denmark.

Pajares, F. (1996). Self-Efficacy beliefs in academic settings. Review of Educational Research, 66(4), 543-578.

Papalia, D.E. (2004). Human Development (9th Edition). New York: McGraw Hill.

Rangel, M.A. (2006). Alimony rights and intrahousehold allocation of resources: Evidence from Brazil. The Economic Journal, 116(513), 627-658.

Republik Indonesia. (2014). Undang-Undang No.35 Tahun 2014 tentang Perubahan atas UndangUndang Nomor 23 Tahun 2002 tentang Perlindungan Anak (Law No.35/2014 revision of UU No.23/2002 about Child Protection).

Smart, J.C. \& Pascarella, E.T. (1986). Self-Concept development and educational degree attainment. Higher Education, 15(1/2), 3-15.

Strauss, J., Witoelar, F., Sikoki, B. \& Wattie, A.M. (2009). The fourth wave of the Indonesia family life survey: Overview and field report volume 1. Santa Monica, CA: RAND Labor and Population.

Teachman, J.D. (1987). Family background, educational resources, and educational attainment. American Sociological Review, 52(4), 548-557.

The United Nation (1989). Child Convention. UN: New York. 